D.M. MOORE, D.H. VALENTINE, S.M. WALTERS y D.A. WEBB [eds.] -1972, 1976, 1980-Flora Europaea. Vols. 3, 4 y 5. Cambridge University Press.

VALDÉS, B., S. TALAVERA y E. FERNÁNDEZGALIANO [eds.] -1987- Flora Vascular de Andalucía Occidental. Ketres Editora S.A. Barcelona.

VELAYOS, M., F. CASTILLA y R. GAMARRA 1991a- Archivos de Flora Ibérica 2 (Corología Ibérica, I). CSIC. Madrid.

VELAYOS, M., F. CASTILLA y R. GAMARRA 1991b-Archivos de Flora Ibérica 3 (Corología Ibérica, II). CSIC. Madrid.

VELAYOS, M., F. CASTILLA y R. GAMARRA 1992- Archivos de Flora Ibérica 5 (Corología Ibérica, III). CSIC. Madrid.
VELAYOS, M. y F. CASTILLA - 1993-Archivos de Flora Ibérica 6 (Corología Ibérica, IV). CSIC. Madrid.

WILLKOMM, M. -1893-Supplementum prodromi Florae Hispanicae. Stuttgart.

WILLKOMM, M. \& J. LANGE -1861-1880Prodromus Florae Hispanicae. 3 tomos. Stuttgart.

Aceptado para su publicación en abril de 2000

Dirección de la autora. Dpto. de Biología Animal, Vegetal y Ecología. Facultad de Ciencias Experimentales. Universidad de Jaén. 23071, JAÉN.

\title{
69. ALGUNOS ELEMENTOS DE INTERÉS EN LA FLORA EDAFÓFILA DE LA PROVINCIA DE GRANADA (S. ESPAÑA)
}

Carlos SALAZAR MENDÍAS, Enrique ARROJO AGUDO, Juan Antonio TORRES, Antonio GARCÍA FUENTES y Francisco VALLE TENDERO

Some interesting elements in the edaphophilous flora of Granada province (S. Spain).

Palabras clave. Citas florísticas, Corología, Ecología.

Keywords. Floristic records, Chorology, Ecology.

Como resultado del estudio de la flora presente tanto en series de vegetación edafohigrófila como en diferentes complejos exoseriales rupícolas y acuáticos de distintas zonas de la provincia de Granada en los últimos años, en este trabajo se comentan ocho táxones de interés ya sea por constituir primeras o segundas citas provinciales o por ser elementos escasos en la Comunidad Autónoma Andaluza que podrían catalogarse como casos de flora amenazada.

Las herborizaciones se han realizado principalmente en los sectores corológicos Subbético, Malacitano-Almijarense y 
Guadiciano-Bacense de la provincia de Granada, según los criterios biogeográficos de Rivas-Martínez et al. (1997).

Para cada taxon se ofrece la localidad en que ha sido hallada, coordenadas UTM y altitud si es posible, fecha de recolección y referencia del pliego depositado en el herbario de la Facultad de Ciencias de la Universidad de Granada (GDAC). Asimismo se aporta un comentario acerca de su corología y ecología junto con algunas observaciones taxonómicas y de estado de conservación en algunos casos.

Aquilegia pyrenaica DC. subsp. cazorlensis (Heywood) Galiano \& Rivas Martínez

GRANADA: Barranco del Buitre. Sierra de Castril. E. Arrojo \& C. Salazar. 24-VII-92, GDAC 43545 .

Endemismo del distrito Cazorlense descrito en 1954 por Heywood en la sierra de Cazorla. Aparece en roquedos umbríos y húmedos del piso oromediterráneo. Nosotros la hemos hallado en las estribaciones de la sierra de Castril (distrito Cazorlense). Anteriormente había sido citada por Valle et al. (1994) sin especificar la localidad y sin aportar el testigo de herbario.

Se trata de una especie catalogada como en peligro de extinción (E) según consta en la legislación internacional, nacional y autonómica. Más recientemente, ha aparecido en la lista roja de plantas amenazadas de la IUCN (Walter \& Gillett, 1998:479) con la categoría de vulnerable (V) en el ámbito tanto local como global.

\section{Oenothera glazioviana Micheli}

GRANADA: Río Darro, VF5317, 890 m. C. Salazar. 7-IX-98, GDAC43546.

Citada por primera vez en la Península Ibérica por Díaz-González \& Navarro Andrés (1978) en comunidades arvenses asturianas. La mayor parte de las citas en el territorio ibérico se localizan en el norte, sobre todo en Galicia, habiendo sido paulatinamente detectada en el centro y oeste peninsular

La hemos hallado naturalizada formando poblaciones extensas en juncales y herbazales higronitrófilos en un tramo de las riberas del río Darro (distrito Alfacarino-Granatense). Constituye por tanto la primera cita para la provincia de Granada y para Andalucía Oriental.

Carum foetidum (Coss. \& Dur.) Benth. \& Hook fil. ex Drude

GRANADA: El Margen (Cúllar), WG3767, 870 m. C. Salazar. 26-X-96, GDAC40662.

Endemismo iberonorteafricano citado en el distrito Guadiciano-Bastetano por Esteve \& Varo (1975). En la actualidad es una planta muy rara y difícil de encontrar, ya que tan sólo hemos hallado un individuo en El Margen de Cúllar. Pensamos que su desaparición puede estar relacionada con la desecación y drenaje de zonas húmedas.

Las localidades más próximas se encuentran en la provincia Murciano-Almeriense, más concretamente en Cartagena, Caravaca, Escombreras y Villena (provincias de Murcia y Alicante) según Ríos et al. (1993). Los saladares de El Margen constituyen la única localidad andaluza en la que se tiene constancia de la presencia de C. foetidum, que siguiendo las categorías de estenocoria de Hernández Bermejo \& Clemente Muñoz (1994) tendría las siglas eE por tratarse de un iberonorteafricanismo y según los criterios actuales de la IUCN la categoría de amenaza local sería en peligro crítico (CR). Consideramos de gran importancia la inclusión de este taxon en la legislación andaluza y la elaboración de planes de recuperación basados en la reintroducción, al tratarse de una especie amenazada de extinción no sólo al nivel provincial o autonómico sino nacional.

\section{Phyla canescens (Kunth.) Greene}

GRANADA: Río Cubillas, VG3825, $610 \mathrm{~m}$. C. Salazar. 28-VIII-98, GDAC43547.

Neófito originario del centro y sur de América que se encuentra naturalizado en el norte de la Península Ibérica, siendo muy frecuentes las citas en Galicia. Fue citado por primera vez en Andalucía por Molesworth Allen (1976:241) en Trebujena (Cádiz) a orillas del río Guadalquivir.

Se trata por tanto de la primera cita provincial de esta especie, concretamente en el distrito Alfacarino-Granatense. Hemos detectado su presencia en prados higrófilos nitrificados (gramales).

Pinguicula dertosensis (Cañig.) Mateo \& Crespo GRANADA: Barranco del Buitre, Sierra de 
Castril. Castril. E. Arrojo \& C. Salazar. 5-VII-93. GDAC43552.

Taxon distribuido de forma dispersa por el este y sureste de la Península Ibérica (Blanca et al., 1999), del que existe constancia de su presencia en la porción granadina del distrito Almijarense (sierras de Tejeda y Almijara) aunque hasta el momento haya sido frecuente su confusión con Pinguicula vallisneriifolia Webb.

Nuestra cita en la sierra de Castril constituye la segunda localidad de esta especie en la provincia de Granada (en este caso en el distrito Cazorlense). La hemos hallado formando parte de comunidades rupícolas con aguas rezumantes carbonatadas.

\section{Centaurea dracunculifolia Dufour}

GRANADA: El Margen (Cúllar), WG3866, 890 m. C. Salazar. 9-VII-94. GDAC40729.

Endemismo iberolevantino que se distribuye en localidades costeras de España y sur de Francia. Fue puesto de manifiesto en la depresión interna de Baza por Esteve \& Varo (1975).

Se trata de una especie que actualmente es extremadamente rara en el distrito GuadicianoBastetano, de la cual se han hallado escasos individuos en juncales halófilos de El Margen de Cúllar. Esta población ha sido prácticamente destruida por las obras de ampliación de una carretera cercana y actualmente se encuentra amenazada por la desecación de los juncales y el exceso de nitrificación debido a la ganadería y las prácticas agrícolas en su entorno. En años recientes apenas se han encontrado individuos reproductores en floración, y se puede considerar que la población está en serio declive.

Es de destacar la importancia de esta especie que alcanza aquí su areal finícola más occidental e interior de la Península Ibérica, siendo la única localidad andaluza en la que se halla presente. Atendiendo a las categorías de estenocoria y amenaza anteriormente referidas, esta planta presentaría las categorías aa y $\mathrm{CR}$ en el territorio andaluz. Consideramos de gran importancia llevar a cabo planes de recuperación de esta especie al borde de la extinción en esta área, basados en un refortalecimiento de la población y reintroducción en otras zonas cercanas.

Cheirolophus intybaceus (Lam.) Dostál GRANADA: Río Verde, Sierra de la Almijara,
VF3479, 850 m. C. Salazar. 19-VIII-98. GDAC43549.

Taxon mediterráneo-occidental termófilo, propio de roquedos calizos. Según Susanna de la Serna (1989) se encuentra ampliamente distribuido por el litoral francés y el Levante español de manera irregular, y en la Península Ibérica penetra hacia el interior por algunos valles.

Fue herborizada en la provincia de Granada por Rivas Goday en 1960 (Susanna de la Serna, op.cit.), concretamente en cerros de Cúllar Baza. Esta es por tanto la segunda cita provincial de un taxon raro, que hemos hallado en una población de escasos individuos situada en un roquedo dolomítico con orientación sur, influenciado por la termicidad del valle del río Verde de Almuñecar (distrito Almijarense),

Galinsoga ciliata (Rafin) S.F. Blake

GRANADA: Río Verde (entre Jete y Almuñecar) VF3868, 40 m. C. Salazar. 3-IX-97. GDAC43551. Río Monachil (Cájar) VG4910, 720 m. C. Salazar. 4-IX-98. GDAC43550.

Neófito procedente del centro y sur de América frecuentemente citado en comunidades arvenses del cuadrante noroeste de la Península Ibérica. Nuestra cita es la primera para Andalucía habiendo sido encontrada a menudo en herbazales higronitrófilos (juncales y herbazales) en las riberas del río Verde (distrito Alpujarreño) y en menor medida en zonas del interior como las del río Monachil cerca de Granada capital (distrito Alfacarino-Granatense).

AGRADECIMIENTOS. Agradecemos a la Dra. Concepción Morales (conservadora del Herbario de la Facultad de Ciencias de la Universidad de Granada) la revisión de los pliegos testigo. Asimismo, agradecemos al Dr. Gabriel Blanca (Universidad de Granada) sus comentarios y la ayuda prestada en la identificación de Centaurea dracunculifolia, Cheirolophus intybaceus y Pinguicula dertosensis.

\section{BIBLIOGRAFÍA}

BLANCA, G.; M. RUIZ-REJÓN \& R. ZAMORA 1999- Taxonomic revision of the genus Pinguicula L. in the Iberian Peninsula. Folia 
Geobotanica 34: 337-361.

DÍAZ-GONZÁLEZ, T.E. y F. NAVARRO ANDRÉS -1978- Las comunidades de Thero-Airion R. Tx. 1951 en las playas asturianas: su posición fitotopográfica. Anales Jard. Bot. Madrid 34(2):571-595.

ESTEVE, F. y J. VARO-1975-Estudio geobotánico de las comunidades halófilas interiores de la provincia de Granada. Anal. Inst. Bot. Cavanilles 32(2):1351-1374.

HERNÁNDEZ-BERMEJO, E. y M. CLEMENTE MUÑOZ -1994- Protección de la Flora Andaluza. Junta de Andalucía. AMA.

MOLESWORTH ALLEN, B. -1976- Notas sobre algunas plantas de la provincia de Cádiz. Lagascalia 6(2):239-242.

RÍOS, S.; A. ROBLEDO; F. ALCARAZ y J. ÁlVAREZ ROGEL -1993- Cuatro plantas de interés para la flora del sureste ibérico. Anales Jard. Bot. Madrid 51(1):162-166.

RIVAS-MARTÍNEZ, S.; A. ASENSI, B. DÍEZGARRETAS, J. MOLERO y F. VALLE -1997Biogeographical syntesis of Andalusia (southern Spain). Journal of Biogeography 24: 915-928. SUSANNA DE LA SERNA, A. -1989-Asientos corológicos para un atlas corológico de la flora occidental, 11. Mapa 114. Fontqueria 22: 1618.

VALLE, F.; E. ARROJO y C. SALAZAR -1994-. Comunidades y táxones de interés ecológico presentes en el Parque Natural de la Sierra de Castril. Monografías de Flora y Vegetación Béticas 7-8: 77-88.

WALTER, K.S. \& H.J. GILLETT (eds.) -1998-. 1997 IUCN Red List of Threatned Plants. IUCN, Gland, Switzerland and Cambridge. Ixiv + 862pp.

Aceptado para su publicación en julio de 2000

Dirección de los autores. C. SALAZAR, J.A. TORRES y A. GARCÍA-FUENTES: Dpto. Biología Animal, Biología Vegetal y Ecología. Facultad de Ciencias Experimentales y de la Salud. Universidad de Jaén, 23071-Jaén.e-mail: csalazar@ujaen.es. F. VALLE y E. ARROJO: Dpto. Biología Vegetal. Facultad de Ciencias. Universidad de Granada. 18001-Granada.

\section{NOTES OF ORCHIDS FROM ANDALUSIA (SPAIN)}

Michael R. LOWE

Notas de Orquideas de Andalucía (España).

Palabras clave. Orquidaceae, Andalucía, España.

Key Words. Orchidaceae, Andalusia, Spain.

As a result of recent field investigations throughout Andalucía, reports are presented of new Provincial records and significant extensions of the known distribution of various orchid species. Particular attention is given to the Genus Epipactis, following recent taxonomic revisions. 